\title{
Interplay between pairing and correlations in spin-polarized bound states
}

\author{
Szczepan Głodzik ${ }^{1}$, Aksel Kobiałka1 ${ }^{1}$ Anna Gorczyca-Goraj ${ }^{2}$, Andrzej Ptok ${ }^{3}$, \\ Grzegorz Górski ${ }^{4}$, Maciej M. Maśka ${ }^{2}$ and Tadeusz Domański ${ }^{* 1}$
}

\author{
Full Research Paper \\ Address: \\ ${ }^{1}$ Institute of Physics, M. Curie-Skłodowska University, 20-031 Lublin, \\ Poland, ${ }^{2}$ Institute of Physics, University of Silesia, 41-500 Chorzów, \\ Poland, ${ }^{3}$ Institute of Nuclear Physics, Polish Academy of Sciences, \\ 31-342 Kraków, Poland and ${ }^{4}$ Faculty of Mathematics and Natural \\ Sciences, University of Rzeszów, 35-310 Rzeszów, Poland \\ Email: \\ Tadeusz Domański* - doman@kft.umcs.lublin.pl \\ * Corresponding author \\ Keywords: \\ bound states in superconductors; Majorana quasiparticles; subgap \\ Kondo effect
}

\begin{abstract}
Beilstein J. Nanotechnol. 2018, 9, 1370-1380.
doi:10.3762/bjnano.9.129
\end{abstract}

Received: 15 December 2017

Accepted: 09 April 2018

Published: 07 May 2018

This article is part of the Thematic Series "Topological materials".

Guest Editor: J. J. Palacios

(C) 2018 Głodzik et al.; licensee Beilstein-Institut.

License and terms: see end of document.

\begin{abstract}
We investigate single and multiple defects embedded in a superconducting host, studying the interplay between the proximity-induced pairing and interactions. We explore the influence of the spin-orbit coupling on energies, polarization and spatial patterns of the bound ( $\mathrm{Yu}-\mathrm{Shiba-Rusinov)} \mathrm{states} \mathrm{of} \mathrm{magnetic} \mathrm{impurities} \mathrm{in} \mathrm{a} \mathrm{two-dimensional} \mathrm{square} \mathrm{lattice.} \mathrm{We} \mathrm{also} \mathrm{address} \mathrm{the} \mathrm{peculiar}$ bound states in the proximitized Rashba chain, resembling the Majorana quasiparticles, focusing on their magnetic polarization that has been recently reported by S. Jeon et al. (Science 2017, 358, 772). Finally, we study leakage of these polarized Majorana quasiparticles into side-attached nanoscopic regions and confront them with the subgap Kondo effect near to the singlet-doublet phase transition.
\end{abstract}

\section{Introduction}

Magnetism is usually detrimental to superconductivity because it breaks the Cooper pairs (at the critical field strength $H_{c 2}$ ). There are, however, a few exceptions in which these phenomena coexist, e.g., in iron pnictides [1], CeCoIn 5 [2]. Also, sometimes magnetic fields induce superconductivity [3]. Plenty of other interesting examples can be found in nanoscopic systems, where magnetic impurities (dots) exhibit a more subtle relationship with the electron pairing driven by the proximity effect $[4,5]$. Cooper pairs easily penetrate the nanoscopic impurities, inducing the bound ( $\mathrm{Yu}-$ Shiba-Rusinov) states that manifest

the local pairing in coexistence with magnetic polarization. Such bound states have been observed in various systems [6-14]. In-gap states (appearing in pairs symmetrically around the Fermi level) can be nowadays controlled electrostatically or magnetically [12] whereas their topography, spatial extent and polarization can be precisely inspected by the state-of-art tunneling measurements $[15,16]$.

It has been reported that adatoms deposited on a twodimensional (2D) superconducting surface develop 
Yu-Shiba-Rusinov (YSR) states, extending to a dozen of intersite distances and they reveal particle-hole oscillations [11]. Bound states of these magnetic impurities in superconducting $\mathrm{NbSe}_{2}$ are characterized by the star shape [17] typical for the rotational symmetry of its triangular lattice. More complex objects, such as dimers, reveal other spatial features, showing the bonding and antibonding states [18]. In a somewhat different context it has been pointed out [19] that exchange coupling between numerous quantum defects involving their intrinsic spins can couple them ferromagnetically. This can be used (e.g., in metallic carbon nanotubes) for a robust transmission of magnetic information over large distances.

In all cases the bound YSR states are also sensitive to interactions. One of them is the spin-orbit coupling (usually meaningful at boundaries, e.g., surfaces) [20-22]. Such interaction in one-dimensional magnetic nanowires can induce the topologically nontrivial superconducting phase, in which the YSR states undergo mutation to Majorana (zero-energy) quasiparticles. Coulomb repulsion between the opposite spin electrons can bring additional important effects. In the proximitized quantum dots it can lead to a parity change (quantum phase transition) with further influence on the subgap Kondo effect (driven by effective spin-exchange coupling with mobile electrons). Furthermore, such spin exchange can be amplified by the induced electron pairing, and can have constructive influence on the Kondo effect $[23,24]$.

We study here the polarized bound states, taking into account the spin-orbit and/or Coulomb interactions. In particular, we consider: (i) a single magnetic impurity in a 2D square lattice of a superconducting host, (ii) a nanoscopic chain of magnetic impurities on the classical superconductor (i.e., proximitized Rashba nanowire) in its topologically trivial/nontrivial superconducting phase, and (iii) a strongly correlated quantum dot side-attached to the Rashba chain, where the Kondo and the leaking Majorana quasiparticle can be confronted with each other. These magnetically polarized YSR and Majorana quasiparticles as well as the subgap Kondo effect can be experimentally verified using tunneling heterostructures with ferromagnetic lead (STM tip).

\section{Results and Discussion Single magnetic impurity}

Let us start by considering a single magnetic impurity on the surface of an $s$-wave superconductor in presence of spin-orbit interactions. This situation can be modeled by the Andersontype Hamiltonian

$$
\widehat{\mathcal{H}}=\widehat{\mathcal{H}}_{\text {sc }}+\widehat{\mathcal{H}}_{\text {imp }}+\widehat{\mathcal{H}}_{\text {SOC }}
$$

We describe the superconducting substrate by

$$
\widehat{\mathcal{H}}_{\mathrm{sc}}=-t \sum_{\langle i, j\rangle \sigma} \hat{c}_{i \sigma}^{\dagger} \hat{c}_{j \sigma}+U \sum_{i} \hat{n}_{i \uparrow} \hat{n}_{i \downarrow}-\mu \sum_{i \sigma} \hat{n}_{i \sigma},
$$

where $\hat{c}_{i \sigma}^{\dagger}\left(\hat{c}_{i \sigma}\right)$ denotes creation (annihilation) of an electron with spin $\sigma$ at the $i$-th site, $t$ is a hopping integral between the nearest neighbors, $\mu$ is the chemical potential, and $\hat{n}_{i \sigma}=\hat{c}_{i \sigma}^{\dagger} \hat{c}_{i \sigma}$ is the number operator. For simplicity, we assume a weak attractive potential $U<0$ between itinerant electrons and treat it within the mean-field decoupling

$$
\begin{aligned}
\hat{c}_{i \uparrow}^{\dagger} \hat{c}_{i \uparrow} \hat{c}_{i \downarrow}^{\dagger} \hat{c}_{i \downarrow} & \approx \chi_{i} \hat{c}_{i \uparrow}^{\dagger} \hat{c}_{i \downarrow}^{\dagger}+\chi_{i}^{*} \hat{c}_{i \downarrow} \hat{c}_{i \uparrow}-\left|\chi_{i}\right|^{2} \\
& +n_{i \uparrow} \hat{c}_{i \downarrow}^{\dagger} \hat{c}_{i \downarrow}+n_{i \downarrow} \hat{c}_{i \uparrow}^{\dagger} \hat{c}_{i \uparrow}-n_{i \uparrow} n_{i \downarrow},
\end{aligned}
$$

where $\chi_{i}=\left\langle\hat{c}_{i \downarrow} \hat{c}_{i \uparrow}\right\rangle$ is the local superconducting order parameter and $n_{i \sigma}=\left\langle\hat{n}_{i \sigma}\right\rangle$. The Hartree term can be incorporated into the local (spin-dependent) chemical potential $\mu \rightarrow \tilde{\mu}_{i \sigma} \equiv \mu-U n_{i \bar{\sigma}}$. The second term in Equation 1 refers to the local impurity

$$
\widehat{\mathcal{H}}_{\mathrm{imp}}=-J\left(\hat{c}_{0 \uparrow}^{\dagger} \hat{c}_{0 \uparrow}-\hat{c}_{0 \downarrow}^{\dagger} \hat{c}_{0 \downarrow}\right)+K\left(\hat{c}_{0 \uparrow}^{\dagger} \hat{c}_{0 \uparrow}+\hat{c}_{0 \downarrow}^{\dagger} \hat{c}_{0 \downarrow}\right)
$$

which affects the order parameter $\chi_{i}$ near the impurity site $i=0$, inducing the YSR states $[25,26]$. In this work we focus on the magnetic term $J$ [4,27], disregarding the potential scattering $K$.

The spin-orbit coupling (SOC) can be expressed by

$$
\widehat{\mathcal{H}}_{\mathrm{SOC}}=-\mathrm{i} \lambda \sum_{i j \sigma \sigma^{\prime}} \hat{c}_{i+\mathbf{d}_{j} \sigma}^{\dagger}\left(\mathbf{d}_{j} \times \hat{\boldsymbol{\sigma}}^{\sigma \sigma^{\prime}}\right) \cdot \widehat{\mathbf{w}}_{i \sigma^{\prime}}
$$

where the vector $\mathbf{d}_{j}=\left(d_{j}^{x}, d_{j}^{y}, 0\right)$ refers to positions of the nearest neighbors of the $i$-th site, and $\hat{\boldsymbol{\sigma}}=\left(\sigma_{x}, \sigma_{y}, \sigma_{z}\right)$ stands for the Pauli matrices. The unit vector $\widehat{\mathbf{w}}$ shows the direction of the spin-orbit field, which can be arbitrary. Here we restrict our considerations to the in-plane $\widehat{\mathbf{w}} \equiv \hat{x}=(1,0,0)$ polarization, which will be important for nontrivial superconductivity in nanowires discussed in the subsection 'Magnetically polarized Majorana quasiparticles'. The other (out-of-plane) component could eventually mix $\uparrow$ and $\downarrow$ spins [22].

Impurities break the translational invariance, therefore the pairing amplitude $\chi_{i}$ and occupancy $n_{i \sigma}$ have to be determined for each lattice site individually. We can diagonalize the Hamiltonian (Equation 1) by the unitary transformation 


$$
\hat{c}_{i \sigma}=\sum_{n}\left(u_{i n \sigma} \hat{\gamma}_{n}-\sigma v_{i n \sigma}^{*} \hat{\gamma}_{n}^{\dagger}\right)
$$

where $\hat{\gamma}_{n}^{(\dagger)}$ are quasiparticle fermionic operators with eigenvectors $u_{i n \sigma}$ and $v_{i n \sigma}$. This leads to the Bogoliubov-de Gennes (BdG) equations

$$
\mathcal{E}_{n}\left(\begin{array}{c}
u_{i n \uparrow} \\
v_{i n \downarrow} \\
u_{i n \downarrow} \\
v_{i n \uparrow}
\end{array}\right)=\sum_{j}\left(\begin{array}{cccc}
H_{i j \uparrow} & D_{i j} & S_{i j}^{\uparrow \downarrow} & 0 \\
D_{i j}^{*} & -H_{i j \downarrow}^{*} & 0 & S_{i j}^{\downarrow \uparrow} \\
S_{i j}^{\downarrow \uparrow} & 0 & H_{i j \downarrow} & D_{i j} \\
0 & S_{i j}^{\uparrow \downarrow} & D_{i j}^{*} & -H_{i j \uparrow}^{*}
\end{array}\right)\left(\begin{array}{c}
u_{j n \uparrow} \\
v_{j n \downarrow} \\
u_{j n \downarrow} \\
v_{j n \uparrow}
\end{array}\right),
$$

where $D_{i j}=\delta_{i j} U \chi_{i}$, and the single-particle term is given by

$$
H_{i j \sigma}=-t \delta_{\langle i, j\rangle}-\left(\tilde{\mu}_{i \sigma}-\sigma J \delta_{i 0}\right) \delta_{i j}+S_{i j}^{\sigma \sigma}
$$

with the spin-orbit coupling term

$$
S_{i j}^{\sigma \sigma^{\prime}}=-\mathrm{i} \lambda \sum_{l}\left(\mathbf{d}_{l} \times \hat{\boldsymbol{\sigma}}^{\sigma \sigma^{\prime}}\right) \cdot \widehat{\mathbf{w}} \delta_{j, i+\mathbf{d}_{l}} .
$$

Here, $S_{i j}^{\sigma \sigma}$ and $S_{i j}^{\sigma \bar{\sigma}}$ (where $\bar{\sigma}$ is opposite to $\sigma$ ) correspond to in-plane and out-of-plane spin-orbit field, respectively, and satisfy $S_{i j}^{\sigma \sigma^{\prime}}=\left(S_{j i}^{\sigma^{\prime} \sigma}\right) *$.

Solving numerically the BdG equations (Equation 6) we can determine the local order parameter $\chi_{i}$ and occupancy $n_{i \sigma}$

$$
\begin{gathered}
\chi_{i}=\sum_{n}\left[u_{i n \downarrow} v_{i n \uparrow}^{*} f\left(\mathcal{E}_{n}\right)-u_{i n \uparrow} v_{i n \downarrow}^{*} f\left(-\mathcal{E}_{n}\right)\right], \\
n_{i \sigma}=\sum_{n}\left[\left|u_{i n \sigma}\right|^{2} f\left(\mathcal{E}_{n}\right)+\left|v_{i n \bar{\sigma}}\right|^{2} f\left(-\mathcal{E}_{n}\right)\right],
\end{gathered}
$$

where $f(\omega)=\left[1+\exp \left(\omega / k_{\mathrm{B}} T\right)\right]^{-1}$. In what follows, we shall inspect the spin-resolved local density of states

$$
\rho_{i \sigma}(\omega)=\sum_{n}\left[\left|u_{i n \sigma}\right|^{2} \delta\left(\omega-\mathcal{E}_{n}\right)+\left|v_{i n \sigma}\right|^{2} \delta\left(\omega+\mathcal{E}_{n}\right)\right] .
$$

For its numerical computation we replace the Dirac delta function with the Lorentzian function $\delta(\omega)=\zeta /\left[\pi\left(\omega^{2}+\zeta^{2}\right)\right]$ with a small broadening $\zeta=0.01 \mathrm{t}$. We have solved the BdG equations, considering a single magnetic impurity in a square lattice, comprising $N_{a} \times N_{b}=41 \times 41$ sites. We assumed $U / t=-3, \mu / t=0$, and determined the bound states for two representative values of the spin-orbit coupling $\lambda$ upon varying $J$.
The magnetic potential has substantial influence on the local order parameter $\chi_{0}$. In particular, at some critical value $J_{\mathrm{c}}$ this quantity discontinuously changes its magnitude and sign (see the upper panel in Figure 1), signaling a first-order phase transition [28-30]. This quantum phase transition at $J_{\mathrm{c}}$ is an artifact of the classical spin approximation. When spin fluctuations are allowed, a Kondo-like crossover is obtained instead of a firstorder phase transition [31,32]. In general, the quasiparticle spectrum at the impurity site is characterized by two bound states $\pm E_{\mathrm{YSR}}$ inside the gap $\Delta$ of the superconducting host (displayed in the bottom panel of Figure 1). These energies $\pm E_{\mathrm{YSR}}$ and the related spectral weights depend on $J$. At $J=J_{\mathrm{c}}$ the YSR bound states cross each other $E_{\mathrm{YSR}}\left(J_{\mathrm{c}}\right)=0$ and their crossing signifies the ground-state parity change [33] from BCS-type (spinless) to the singly occupied (spinful) configurations $[8,15,21,34]$. Let us remark that this quantum phase transition is also accompanied with a reversal of the YSR polarization (see bottom panel in Figure 1). A similar behavior can be observed also for multiple impurities, at several critical values of $J[35]$.

Within the BdG approach we can inspect spatial profiles of the YSR states by integrating the spectral weights

$$
\rho_{i \sigma}^{ \pm}=\int_{\omega_{1}}^{\omega_{2}} \rho_{i \sigma}(\omega) \mathrm{d} \omega
$$

in the interval $\omega \in\left(\omega_{1}, \omega_{2}\right)$ capturing the quasiparticles at negative/positive energies $\pm E_{\mathrm{YSR}}$ [36]. Figure 2 illustrates the results obtained for $\lambda=0$ (left panel) and $\lambda=t$ (right panel). We clearly notice a fourfold rotational symmetry (typical for the square lattice) and the spatial extent of YSR states reaching several sites away from the magnetic impurity. The non-vanishing difference of the spectral weight $\left|u_{i n \uparrow}\right|^{2}-\left|u_{i n \downarrow}\right|^{2}$ at the positive energy $\omega=+E_{Y S R}$ and of $\left|v_{i n \uparrow}\right|^{2}-\left|v_{i n \downarrow}\right|^{2}$ at the negative energy $\omega=-E_{Y S R}$ implies the effective spin-polarization of the bound states (their polarization is illustrated in the bottom panel of Figure 1).

For a quantitative estimation of the spatially varying magnetization (driven by the particle-hole asymmetry) we have computed the displaced moving average $\bar{\rho}^{ \pm}(r)$, which corresponds to an averaged spectral weight contained in a ring of the radius $r$ and a small half-width $\delta r$. This quantity is sensitive only to the radial distance $r$ from the magnetic impurity, averaging the angular anisotropy. Our results, presented in Figure 3, clearly indicate the spatial particle-hole oscillations $\bar{\rho}^{ \pm}(r)$ of the YSR states (compare the blue and red lines). Such particle-hole oscillations decay exponentially with $r$ in agreement with previous studies $[11,37,38]$. The dominant (particle or hole) contributions to the YSR bound states are displayed by the 

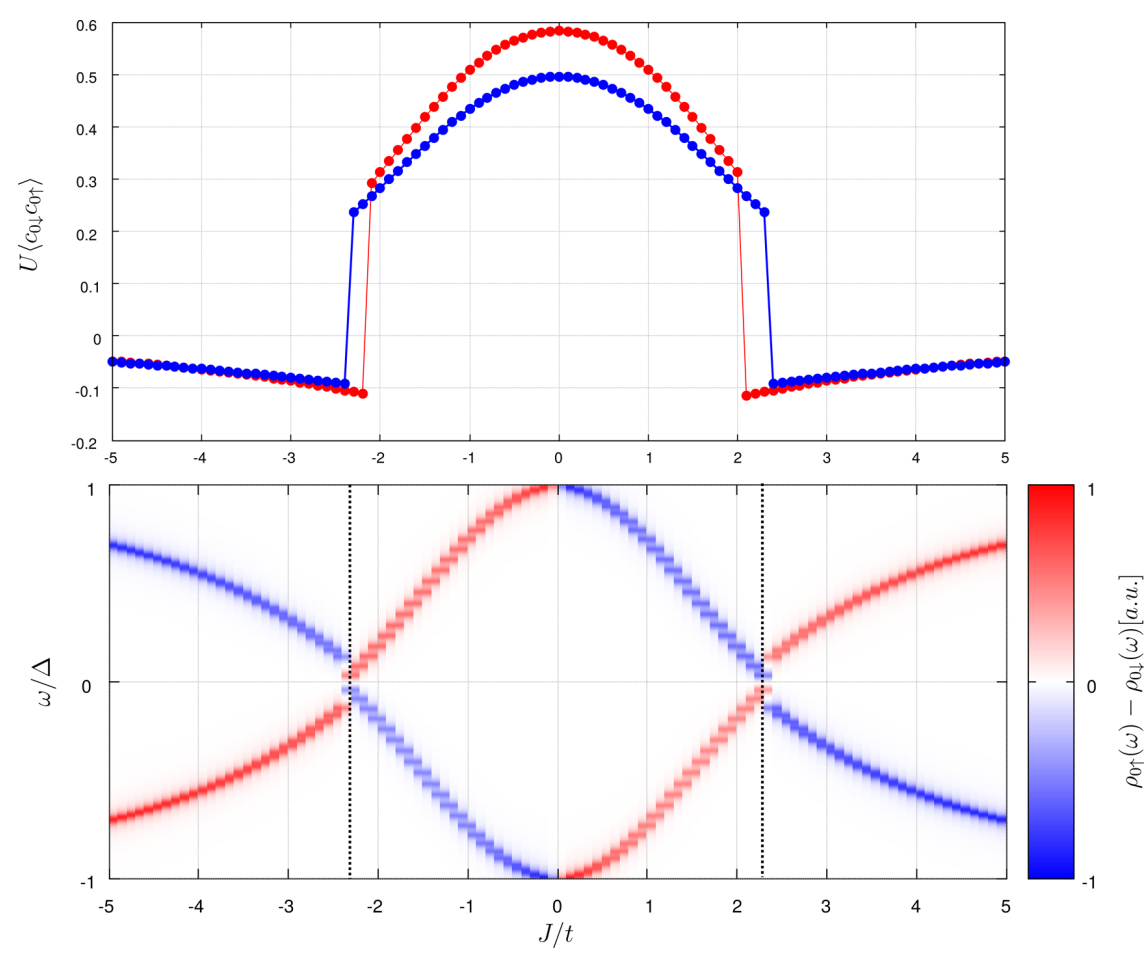

Figure 1: The local order parameter obtained at zero temperature for weak $\lambda / t=0.1$ (red line) and strong spin-orbit coupling $\lambda / t=1$ (blue line). The bottom panel shows the energies and magnetic polarization $\rho_{0 \uparrow}(\omega)-\rho_{0 \downarrow}(\omega)$ of $Y S R$ states obtained in the weak-coupling limit $\lambda / t=0.1$.

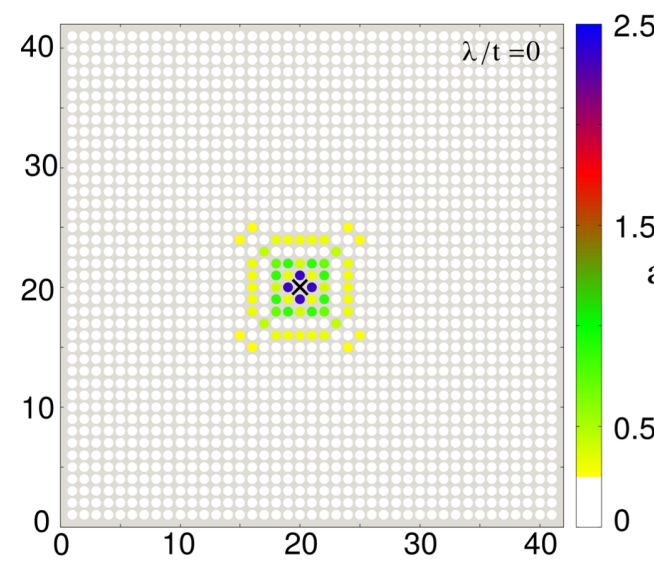

1.5

a.u.

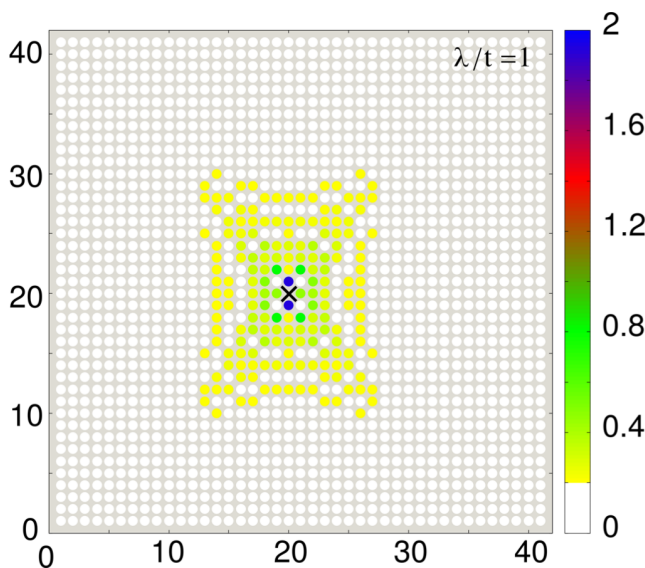

Figure 2: Spatial profiles of the YSR states $\sum_{\sigma} \rho_{i \sigma}^{+}$obtained for $|J|<J_{C}$ in the absence of spin-orbit coupling (left panel) and for strong in-plane coupling $\lambda=t$ (right panel). The spin-orbit field is chosen along the $x$-axis and leads to an additional imaginary hopping term along the $y$-axis, which elongates the YSR states in the $y$-direction. The impurity spin is oriented along the $(0,0,1)$ direction.

alternating color of the background in Figure 3. We notice that the spin-orbit coupling seems to suppress these particle-hole oscillations.

Summarizing this section, we point out that the quantum phase transition at $J_{\mathrm{c}}$ depends on the spin-orbit coupling $\lambda$ and it has experimentally observable consequences in the magnetization induced near the impurity site. For weak magnetic scattering
$|J|<J_{\mathrm{c}}$ the impurity is partly screened, whereas for stronger couplings $|J|>J_{\mathrm{c}}$ the impurity polarizes its neighborhood in the direction of its own magnetic moment. Similar effects have been previously discussed in [21], but here we additionally consider the role of spin-orbit coupling. First of all, such interaction shifts the quantum phase transition (to larger values of $J$ ) and secondly it enhances the spatial extent of YSR states and gradually smoothes the particle-hole oscillations. 

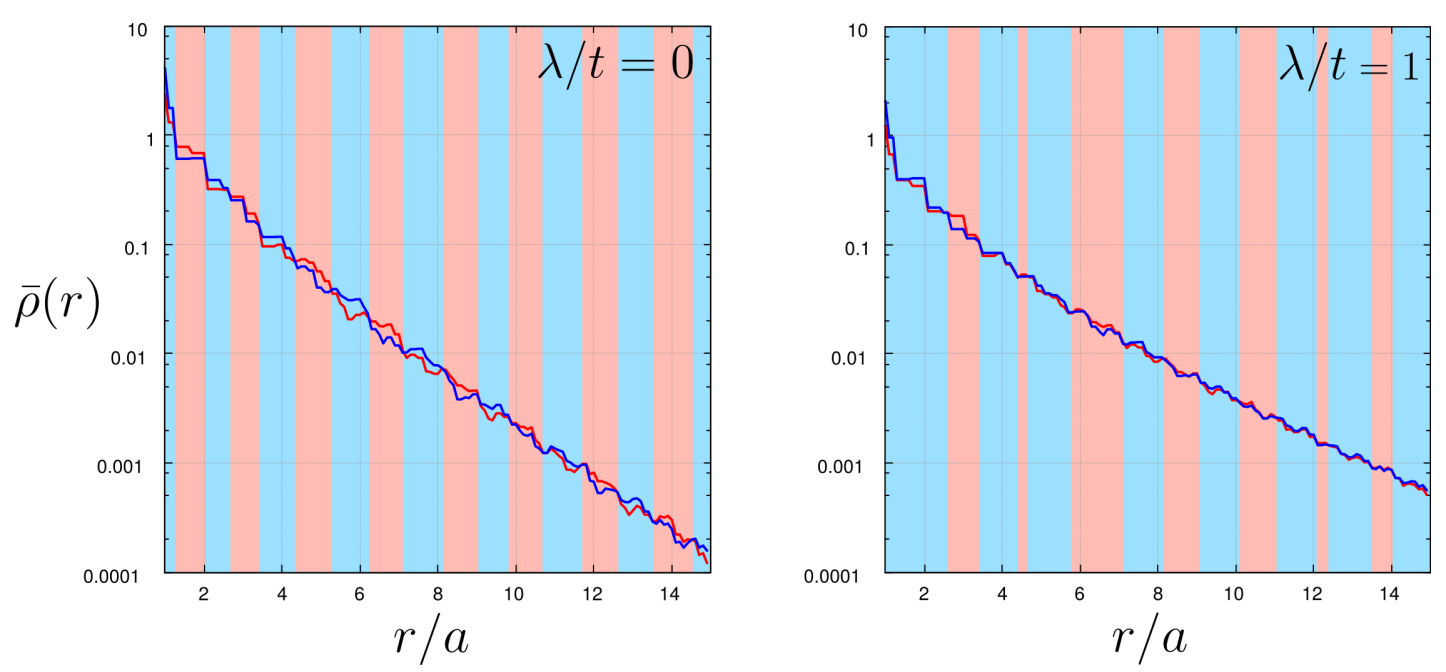

Figure 3: Hole-like (blue line) and electron-like (red line) displaced moving average $\bar{\rho}^{ \pm}(r)$ as a function of the radial distance $r$ from the impurity site obtained for $|J|<J_{C}$ using $\delta r=0.5 a$. The blue and red background color indicates the dominant type (hole or particle) of the YSR states at a given distance $r$. The left and right panels correspond to $\lambda=0$ and $\lambda=t$, respectively.

\section{Magnetically polarized Majorana quasiparti- cles}

In this section we increase the number of impurities. Let us now imagine a nanoscopic chain of magnetic impurities (for instance Fe atoms) deposited on the surface of a conventional $s$-wave superconductor. We study the magnetically polarized bound states, focusing on the proximity-induced nontrivial superconducting phase. In practice, the quasiparticle spectrum can be probed within STM-type setups, by attaching a conducting $[39,40]$, superconducting [41], or a magnetically polarized tip [42]. We assume the spin-orbit interaction aligned perpendicularly to the wire and the magnetic field parallel to it, leading to the effective intersite pairing of identical spins and (under specific conditions) inducing zero-energy end modes resembling Majorana quasiparticles. This issue has been recently studied very intensively but here we simply focus on the spin-polarized aspects of this problem.

Due to the spin-orbit interaction, momentum and spin are no longer "good" quantum numbers. By solving the problem numerically, however, we can estimate the percentage with which the true quasiparticles are represented by the initial spin. We have recently emphasized [43], that the amplitude of intersite pairing (between identical spin electrons) differs several times for $\uparrow$ and $\downarrow$ sectors. This leads to an obvious polarization of the YSR and Majorana quasiparticles (the latter appearing near the nanochain edges).

Let us consider the STM-type geometry relevant to the recent experimental situation addressed by A. Yazdani and co-workers [42], which can be described by the following Hamiltonian

$$
\widehat{\mathcal{H}}=\widehat{\mathcal{H}}_{\text {tip }}+\widehat{\mathcal{H}}_{\text {chain }}^{\text {prox }}+\widehat{\mathcal{H}}_{\text {tip-chain }}
$$

We assume here that the STM tip describes a polarized fermion gas

$$
\widehat{\mathcal{H}}_{\mathrm{N}}=\sum_{\mathbf{k}, \sigma} \xi_{\mathbf{k N}}^{\sigma} \hat{c}_{\mathbf{k} \sigma \mathrm{N}}^{\dagger} \hat{c}_{\mathbf{k} \sigma \mathrm{N}}
$$

where the energy $\xi_{\mathbf{k N}}^{\sigma}=\varepsilon_{\mathbf{k}}-\mu_{\mathrm{N \sigma}}$ can be controlled by some finite detuning of the chemical potentials $\mu_{\mathrm{N} \uparrow}-\mu_{\mathrm{N} \downarrow}$. Individual atoms of the nanochain are coupled with such STM tip through

$$
\widehat{\mathcal{H}}_{\text {tip-chain }}=\sum_{\mathbf{k}, \sigma}\left(V_{i, \mathbf{k N}} \hat{d}_{i, \sigma}^{\dagger} \hat{c}_{\mathbf{k} \sigma \mathrm{N}}+V_{i, \mathbf{k} \beta}^{*} \hat{c}_{\mathbf{k} \sigma \mathrm{N}}^{\dagger} \hat{d}_{i, \sigma}\right) .
$$

For simplicity, we assume constant couplings

$$
\Gamma_{\beta}=2 \pi \sum_{\mathbf{k}}\left|V_{i, \mathbf{k} \beta}\right|^{2} \delta\left(\omega-\xi_{\mathbf{k} \beta}\right)
$$

The low-energy physics of such proximitized Rashba nanowire can be described by [44]

$\widehat{\mathcal{H}}_{\text {chain }}^{\text {prox }}=\sum_{i, j, \sigma}\left(t_{i j}-\delta_{i j} \mu\right) \hat{d}_{i, \sigma}^{\dagger} \hat{d}_{j, \sigma}+\widehat{\mathcal{H}}_{\text {Rashba }}+\widehat{\mathcal{H}}_{\text {Zeeman }}+\widehat{\mathcal{H}}_{\text {prox }}$,

where $\hat{d}_{i, \sigma}^{(\dagger)}$ annihilates (creates) an electron of spin $\sigma$ at site $i$ with energy $\varepsilon_{i}$, and $t_{i j}$ is the hopping integral. The effective intersite ( $p$-wave) pairing is induced through a combined effect of the Rashba and the Zeeman terms 


$$
\begin{gathered}
\widehat{\mathcal{H}}_{\text {Rashba }}=-\alpha \sum_{i, \sigma, \sigma^{\prime}}\left[\hat{d}_{i+1, \sigma}^{\dagger}\left(i \sigma^{y}\right)_{\sigma \sigma^{\prime}} \hat{d}_{i, \sigma^{\prime}}+\text { H.c. }\right] \\
\widehat{\mathcal{H}}_{\text {Zeeman }}=\frac{g \mu_{\mathrm{B}} B}{2} \sum_{i, \sigma, \sigma^{\prime}} \hat{d}_{i, \sigma}^{\dagger}\left(\sigma^{z}\right)_{\sigma \sigma^{\prime}} \hat{d}_{i, \sigma^{\prime}} .
\end{gathered}
$$

The proximity effect, which induces the on-site (trivial) pairing, can be modelled as [45]

$$
\widehat{\mathcal{H}}_{\text {prox }}=\Delta_{i}\left(\hat{d}_{i, \uparrow}^{\dagger} \hat{d}_{i, \downarrow}^{\dagger}+\hat{d}_{i, \downarrow} \hat{d}_{i, \uparrow}\right)
$$

with the local pairing potential $\Delta_{i}=\Gamma_{\mathrm{S}} / 2$.

Figure 4 shows evolution of the spin-dependent spectrum $\rho_{i \sigma}(\omega)$ as a function of a varying magnetic field. At a critical value ( $B \approx 0.2$ ) we observe the emergence of zero-energy quasiparticles, whose spectral weights strongly depend on the spin $\sigma$.
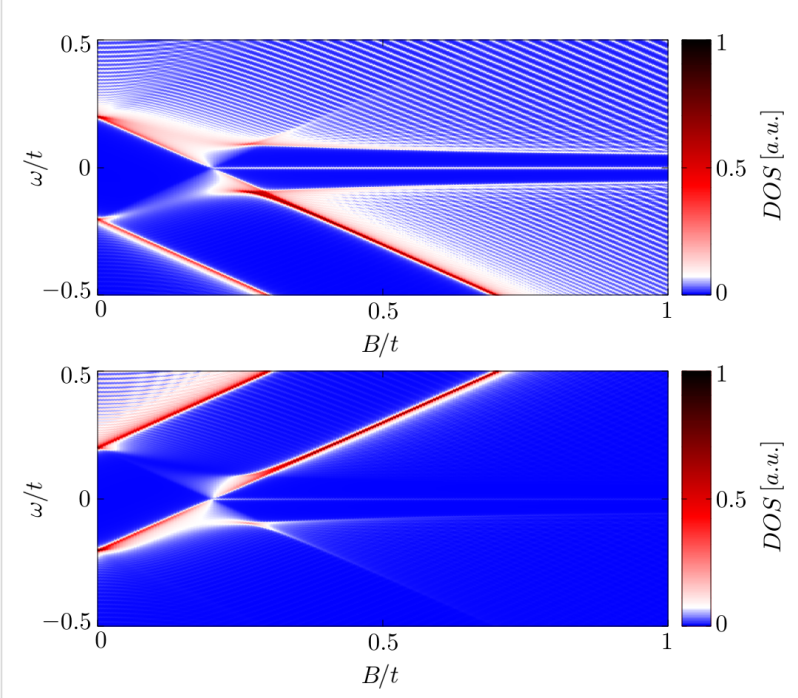

Figure 4: The effective quasiparticle spectrum $\rho_{i \sigma}(\omega)$ as a function of a magnetic field $B$ aligned along the nanochain obtained for $\sigma=\uparrow$ (upper panel) and $\sigma=\downarrow$ (bottom panel). The magnetic field $B$ is expressed in units of $t /\left(g \mu_{\mathrm{B}} / 2\right)$.

For a better understanding of the polarized zero-energy quasiparticles, we present in Figure 5 the spatial profiles of the zeroenergy (Majorana) quasiparticles. As usually such quasiparticles emerge near the edges of a nanoscopic chain, practically over 10 to 15 sites (see inset). Note the substantial quantitative difference between these zero-energy quasiparticles appearing in $\uparrow$ and $\downarrow$ spin sectors. This "intrinsic polarization" of the Majorana modes has been previously suggested in [46], and recently we have proposed [47] their empirical detection by means of selective equal-spin Andreev reflection (SESAR) spectroscopy.

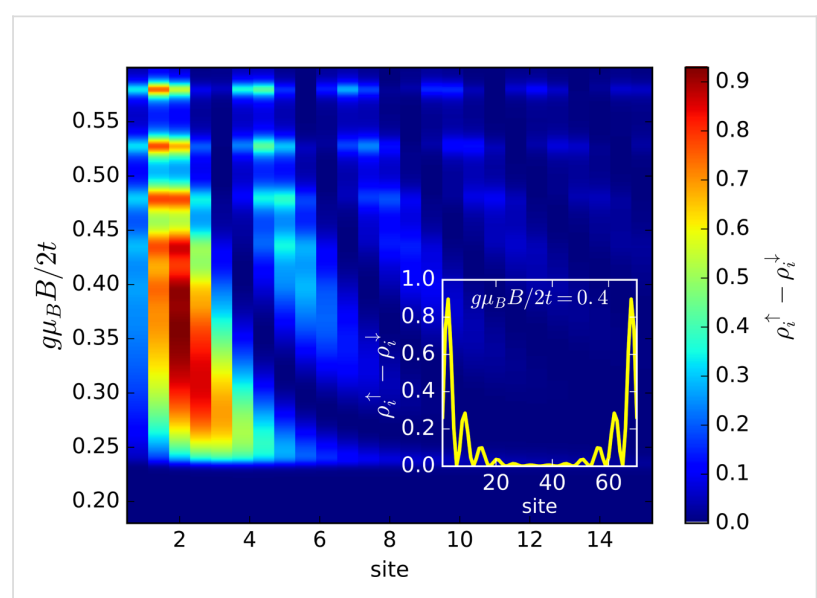

Figure 5: Magnetically polarized spectrum $\rho_{i, \uparrow}(\omega)-\rho_{i, \downarrow}(\omega)$ obtained at $\omega=0$ for peripheral sites of the Rashba chain.

The main idea is to apply a bias voltage $V$ between the STM tip and the superconducting substrate, inducing a charge transport that, in a subgap regime $(|V| \ll \Delta /|e|)$ originates from the Andreev (particle to hole) scattering mechanism. The polarized Andreev current can be expressed by the Landauer-Büttiker formula

$$
I_{i}^{\sigma}(V)=\frac{e}{h} \int \mathrm{d} \omega T_{i}^{\sigma}(\omega)[f(\omega-e V)-f(\omega+e V)]
$$

where transmittance is defined as

$$
T_{i}^{\sigma}(\omega)=\Gamma_{\mathrm{N}}^{2}\left|\left\langle\left\langle\hat{d}_{i \sigma} \hat{d}_{i+1 \sigma}\right\rangle\right\rangle\right|^{2}+\Gamma_{\mathrm{N}}^{2}\left|\left\langle\left\langle\hat{d}_{i \sigma} \hat{d}_{i-1 \sigma}\right\rangle\right\rangle\right|^{2}
$$

and

$$
\begin{aligned}
& T_{1}^{\sigma}(\omega)=\Gamma_{\mathrm{N}}^{2}\left|\left\langle\left\langle\hat{d}_{1 \sigma} \hat{d}_{2 \sigma}\right\rangle\right\rangle\right|^{2}, \\
& T_{\mathrm{N}}^{\sigma}(\omega)=\Gamma_{\mathrm{N}}^{2} \mid\left\langle\left.\left\langle\hat{d}_{N \sigma} \hat{d}_{N-1 \sigma}\right\rangle\right|^{2} .\right.
\end{aligned}
$$

The anomalous Green's functions can be computed numerically from the solution of the Bogoliubov-de Gennes equations of this model (Equation 10). The net spin current $I_{i}^{\text {spin }}(V)=I_{i}^{\uparrow}(V)-I_{i}^{\downarrow}(V)$ turns out to be predominantly sensitive to the Majorana end-modes. Its differential conductance $G_{i}^{\text {spin }}(V)=(\partial / \partial V) I_{i}^{\text {spin }}(V)$ can thus distinguish the polarized Majorana quasiparticle (near $V=0$ ) from the YSR states (appearing at finite voltage). 
Bound states can leak to other side-attached nanoscopic objects. This proximity effect has been also predicted for the Majorana quasiparticles by E. Vernek et al. [48] and it has been indeed observed experimentally by M. T. Deng and co-authors [49]. Inspired by this achievement, extensive studies have been carried out regarding the YSR states coalescencing into the zero-energy Majorana states in side-coupled quantum dots driven by electrostatic or magnetic fields [50-52]. This issue would be particularly important when attempting to braid the Majorana end modes, e.g., in T-shape nanowires upon turning on and off the topological superconducting phase in its segments. We briefly analyse here the polarized zero-energy Majorana modes leaking into the multi-site quantum dot (comprising ten lattice sites) side-attached to the proximitized Rashba chain discussed above.

Figure 6 displays the spatial profile of the polarized spectrum obtained at $\omega=0$ as a function of the gate voltage $V_{\mathrm{g}}$, which detunes the energies $V_{\mathrm{g}}=\varepsilon_{i}-\mu$ of the multi-site $(1 \leq i \leq 10)$ quantum dot. For numerical calculations we used the model parameters $\lambda=0.15 t, \mu=-2 t, \Delta_{i}=0.2 t$ and $B>B_{\mathrm{c}}$, which guarantee the Rashba chain to be in its topologically nontrivial superconducting phase, hosting the zero-energy Majorana quasiparticles (intensive black or red regions). We clearly observe that for some values of $V_{\mathrm{g}}$ these Majorana modes spread over the entire quantum dot region. By inspecting Figure 6 we furthermore notice the pronounced spatial oscillations of these zero-energy modes. In our opinion, this is a signature of a partial delocalization of the polarized Majorana quasiparticles. Surprisingly, this process seems to be less efficient in
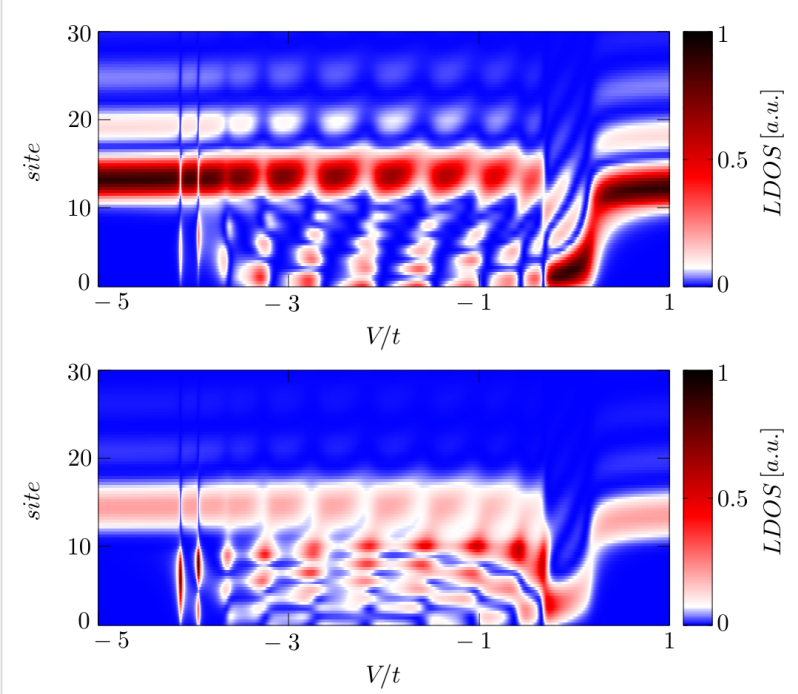

Figure 6: Leakage of the spin-polarized Majorana quasiparticles from the topological superconducting phase of the Rashba chain $(i \geq 10)$ onto the side-attached multi-site $(i \in\langle 1 ; 10\rangle)$ quantum dot. The upper and bottom panel show $\rho_{i \sigma}(\omega)$ at $\omega=0$ for $\uparrow$ and $\downarrow$ spin, respectively. the minor spin $(\sigma=\downarrow)$ section. This effect has to be taken into account, when designing nanostructures for a controllable spatial displacement of the Majorana modes (criticial for the realization of quantum computations with use of the Majoranabased qubits) either by electrostatic or magnetic means. Some proposals for such nanodevices have been recently discussed by several authors [52,53].

In summary of this section, we emphasize that the Majorana modes coalescing from the YSR states in the proximitized Rashba nanowire are characterized by their magnetic polarization. Indeed, such a feature has been recently observed by STM spectroscopy with use of a polarized tip [42]. We have studied here the evolution of the polarized quasiparticle states with respect to the magnetic field (Figure 4) and investigated the spatial oscillations of the Majorana zero-energy modes near the chain edges (Figure 5). Finally, we analyzed leakage of the polarized Majorana modes on the multi-site quantum dots, revealing their partial delocalization (Figure 6).

\section{Majorana vs Kondo effect}

In previous section we have discussed the polarized Majorana modes leaking into side-attached objects, such as single impurities or segments of normal nanowires. In this section we shall focus on the correlation effects [54-56], confronting the Majorana quasiparticle with the Kondo effect (both manifested at zero energy). This can be practically achieved using STM-type configurations sketched in Figure 7. In particular, we consider the subgap Kondo effect, effectively driven by the Coulomb repulsion $U$ and coupling of the quantum dot (QD) with the normal lead $\Gamma_{\mathrm{N}}$ in presence of electron pairing (induced via $\Gamma_{\mathrm{S}}$ ), which has a significant influence on the spin-polarized bound states of the QD. The basic mechanism of this subgap Kondo effect showing up near the quantum phase transition has been earlier considered by us in absence of the Rashba nanowire

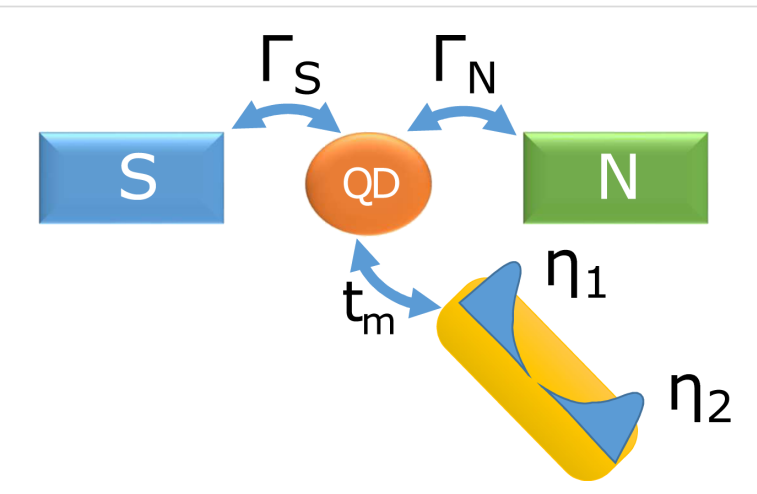

Figure 7: Schematic illustration of the quantum dot (QD) coupled between the metallic $(\mathrm{N})$ and superconducting $(\mathrm{S})$ leads and hybridized with the Rashba nanowire, hosting the Majorana quasiparticles $\eta_{1}$ and $\eta_{2}$ at its edges. 
[24,57]. Our considerations can be practically verified within STM geometry $[39,40]$ using magnetic atoms (e.g., Fe) and side-coupled nonmagnetic atoms (for instance $\mathrm{Ag}$ or $\mathrm{Au}$ ) deposited on the superconducting substrate (such as $\mathrm{Pb}$ or $\mathrm{Al}$ ) probed with a conducting STM tip [42].

The topological superconducting phase, hosting the Majorana modes, can be driven in semiconducting wires [58,59] or in nanochains of magnetic atoms [39-42] through nearest-neighbor equal-spin pairing. The efficiency of such $p$-wave pairing differs for each spin [47], giving rise to polarization of the Majorana quasiparticles, with noticeable preference for the $\uparrow$ sector (see Figure 4). In order to study the correlation effects we shall assume here a complete polarization of the Majorana quasiparticles. We thus focus, for simplicity, on the topological state originating from intersite pairing of only $\uparrow$ electrons and consider its interplay with the correlations. Let us remark, however, that the superconducting lead mixes both the QD spins with the side-attached Majorana quasiparticle [60]. In consequence we shall observe an interesting and spin-dependent relationship between the Majorana and Kondo states that could be probed by the polarized Andreev (particle-to-hole conversion) mechanism.

Our setup (Figure 7) can be described by the following Anderson-type Hamiltonian

$$
\widehat{\mathcal{H}}=\sum_{\beta=\mathrm{S}, \mathrm{N}}\left(\widehat{\mathcal{H}}_{\beta}+\widehat{\mathcal{H}}_{\beta-\mathrm{QD}}\right)+\widehat{\mathcal{H}}_{\mathrm{QD}}+\widehat{\mathcal{H}}_{\mathrm{MQD}}
$$

where $\widehat{\mathcal{H}}_{\mathrm{N}}$ corresponds to the metallic electrode, $\widehat{\mathcal{H}}_{\mathrm{S}}$ refers to the $s$-wave superconducting substrate and the correlated QD is modeled by $\widehat{\mathcal{H}}_{\mathrm{QD}}=\sum_{\sigma} \varepsilon \hat{d}_{\sigma}^{\dagger} \hat{d}_{\sigma}+U \hat{n}_{\downarrow} \hat{n}_{\uparrow}$, where $\varepsilon$ denotes the energy level and $U$ stands for the repulsive interaction between opposite spin electrons. The QD is coupled to both $\beta=\mathrm{N}, \mathrm{S}$ reservoirs through $\widehat{\mathcal{H}}_{\beta-\mathrm{QD}}=\sum_{\mathbf{k}, \sigma}\left(V_{\mathbf{k} \beta} \hat{d}_{\sigma}^{\dagger} \hat{c}_{\mathbf{k} \sigma \beta}+\right.$ H.c. $)$ and we assume a wide bandwidth limit, using the constant couplings $\Gamma_{\beta}$. It can be shown [61-64] that for energies $|\omega| \ll \Delta$ the super-conducting electrode induces the static on-dot pairing

$$
\begin{aligned}
\widehat{\mathcal{H}}_{\mathrm{S}}+\widehat{\mathcal{H}}_{\mathrm{S}-\mathrm{QD}} \approx \mathcal{H}_{\text {prox }}= & \sum_{\sigma} \varepsilon \hat{d}_{\sigma}^{\dagger} \hat{d}_{\sigma}+U \hat{n}_{\downarrow} \hat{n}_{\uparrow} \\
& -\frac{\Gamma_{\mathrm{S}}}{2}\left(\hat{d}_{\uparrow} \hat{d}_{\downarrow}+\hat{d}_{\downarrow}^{\dagger} \hat{d}_{\uparrow}^{\dagger}\right) .
\end{aligned}
$$

Taking into account the finite magnitude of superconducting gap [50] does not affect the main conclusions of our study.

The effective Majorana modes of the nanowire can be modeled by [65]

$$
\widehat{\mathcal{H}}_{\mathrm{MQD}}=\mathrm{i} \varepsilon_{m} \hat{\eta}_{1} \hat{\eta}_{2}+\lambda\left(\hat{d}_{\uparrow} \hat{\eta}_{1}+\hat{\eta}_{1} \hat{d}_{\uparrow}^{\dagger}\right)
$$

where $\hat{\eta}_{i}=\hat{\eta}_{i}^{\dagger}$ are Hermitian operators and $\varepsilon_{m}$ corresponds to an overlap between Majoranas. We recast these operators by the standard fermionic ones [66] $\hat{\eta}_{1}=(1 / \sqrt{2})\left(\hat{f}+\hat{f}^{\dagger}\right)$ and $\hat{\eta}_{2}=(-\mathrm{i} / \sqrt{2})\left(\hat{f}-\hat{f}^{\dagger}\right)$. Finally, the Hamiltonian of Equation 15 simplifies to

$$
\begin{aligned}
\widehat{\mathcal{H}}= & \widehat{\mathcal{H}}_{\mathrm{N}}+\widehat{\mathcal{H}}_{\mathrm{N}-\mathrm{QD}}+\sum_{\sigma} \varepsilon \hat{d}_{\sigma}^{\dagger} \hat{d}_{\sigma}+U \hat{n}_{\downarrow} \hat{n}_{\uparrow}-\frac{\Gamma_{S}}{2}\left(\hat{d}_{\uparrow} \hat{d}_{\downarrow}+\hat{d}_{\downarrow}^{\dagger} \hat{d}_{\uparrow}^{\dagger}\right) \\
& +\varepsilon_{m} \hat{f}^{\dagger} \hat{f}+t_{m}\left(\hat{d}_{\uparrow}^{\dagger}-\hat{d}_{\uparrow}\right)\left(\hat{f}+\hat{f}^{\dagger}\right)-\frac{\varepsilon_{m}}{2},
\end{aligned}
$$

with the auxiliary coupling $t_{m}=\lambda / \sqrt{2}$. The subgap Kondo physics originates in this model from the Coulomb term $U \hat{n}_{\downarrow} \hat{n}_{\uparrow}$ and the effective spin-exchange interactions due to $\widehat{\mathcal{H}}_{\mathrm{N}-\mathrm{QD}}$. It has been shown $[23,24]$ that under specific conditions the on-dot pairing can cooperate with the subgap Kondo effect. This particular situation occurs only near the quantum phase transition.

Let us examine how the subgap Kondo effect gets along with the Majorana mode. Earlier studies of the correlated quantum dot coupled to both normal (conducting) electrodes indicated that the side-attached Rashba chain leads to a competition between the Kondo and Majorana states [67-72]. For a sufficiently long wire $\left(\varepsilon_{m}=0\right)$ the Kondo effect persists only in the spin-channel $\downarrow$, whereas for $\uparrow$ electrons there appears a dip in the spectral density at $\omega=0$. The resulting tunneling conductance is then partly reduced (from the perfect value $2 e^{2} / h$ ) to the fractional value $3 e^{2} / 2 h[67,68,71-73]$. In contrast, for the short Rashba wires (with $\varepsilon_{m} \neq 0$ ) the Kondo physics persists in both spin channels.

In our present setup (Figure 7) the correlated quantum dot is between the metallic and superconducting reservoirs, therefore the Kondo effect is additionally affected by on-dot pairing. Its influence is mainly controlled by the ratio $U / \Gamma_{\mathrm{S}}$ and partly by the level $\varepsilon$, determining whether the QD ground state is in the spinful or spinless configuration $[23,24,62,64,74]$. Obviously the latter one cannot be screened. For instance, for the halffilled QD $(\varepsilon=-U / 2)$ the spinful (doublet) configuration occurs in the regime $U \geq \Gamma_{\mathrm{S}}$.

For studying the correlations we adopt perturbative treatment of the Coulomb potential, treating it self-consistently to the second order in the normal and anomalous channels $[62,75]$. Specific expressions have been provided by us in [24]. Figure 8 shows the spectral function $\rho_{\sigma}(\omega)$ for both spins obtained at zero temperature for the Coulomb potential $U$, covering the (spinless) 
singlet and (spinful) doublet configurations. In the weak interaction regime we observe appearance of two YSR states. For $U \approx \Gamma_{\mathrm{S}}$ these peaks merge, signaling the quantum phase transition. The Kondo effect shows up only in the correlated limit $\left(U>\Gamma_{\mathrm{S}}\right)$, but its spectroscopic signatures are qualitatively different for each of the spins. Leakage of the Majorana quasiparticle suppresses the low-energy states of $\uparrow$ electrons. We notice that the initial density (for $t_{m}=0$ ) is reduced by half, whereas we observe a constructive influence of the Majorana quasiparticle on opposite-spin $\downarrow$ electrons.

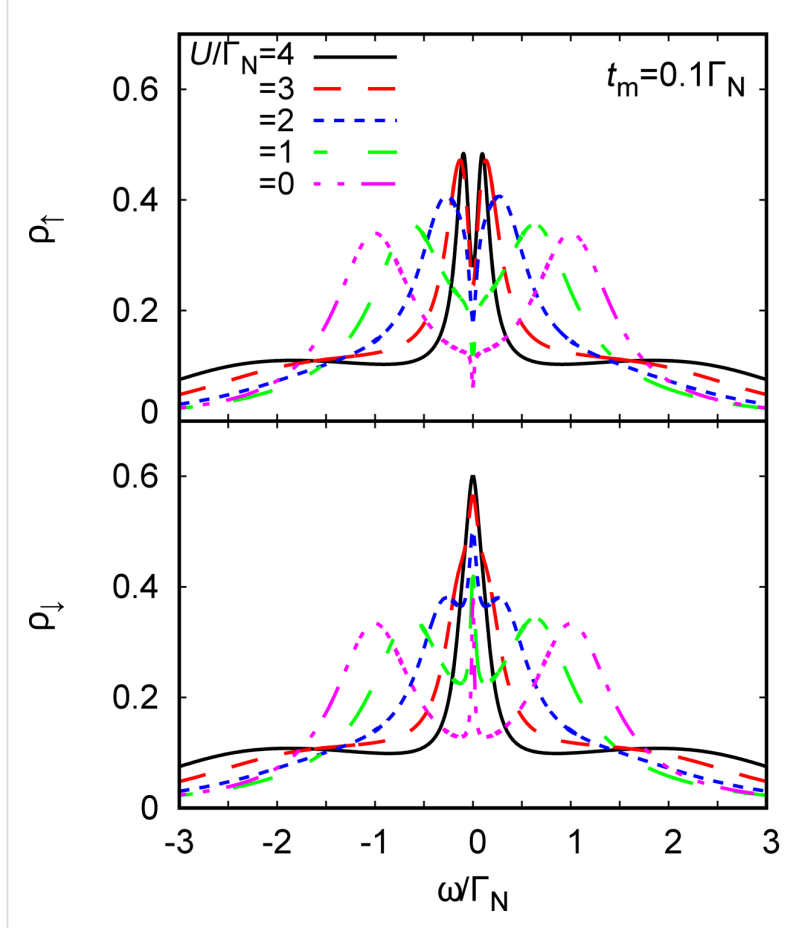

Figure 8: The polarized spectral function $\rho_{\sigma}(\omega)$ obtained at zero temperature for the half-filled QD $(\varepsilon=-U / 2), \Gamma_{S}=2 \Gamma_{N}, t_{m}=0.1 \Gamma_{N}$ and several values of the Coulomb potential $U$ (as indicated). Energies are expressed in units of $\Gamma_{\mathrm{N}}$.

Figure 9 shows evolution of the spectral function $\rho_{\uparrow}(\omega)$ for various couplings $t_{m}$. In the weak-coupling limit we clearly observe a reduction (by half) of the initial density of states. With increasing $t_{m}$ the spectrum develops the three-peak structure that is typical for the "molecular" limit. This behavior indicates that the Majorana and Kondo states have rather a complicated relation, which is neither competitive nor cooperative. In fact, some novel scaling laws have been recently reported by several authors [69,70,76-79] also considering the correlation effects directly in the Rashba nanowire.

\section{Conclusion}

We have studied the polarized bound states of magnetic impurities embedded in an $s$-wave superconducting material, taking

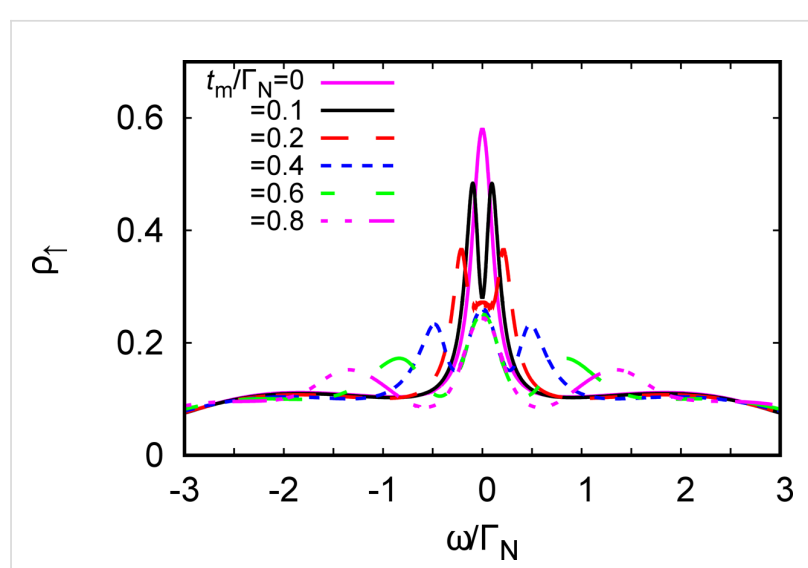

Figure 9: The spectral function $\rho_{\uparrow}(\omega)$ of the half-filled quantum dot $(\varepsilon=-U / 2)$ obtained at $T=0$ for $\Gamma_{S} / \Gamma_{N}=2, U / \Gamma_{N}=4$ and several values of $t_{m}$ (as indicated).

into account the spin-orbit and/or Coulomb interactions. We have shown that spin-orbit coupling strongly affects the subgap states, both of the single impurities and their conglomerate arranged into a nanoscopic chain. For the case of single magnetic impurity the spin-orbit interaction (i) shifts the quantum phase transition towards higher magnetic coupling $J_{\mathrm{c}}$, (ii) enhances the spatial size of the YSR states, and (iii) smoothes the particle-hole oscillations. For the magnetic chain spin-orbit coupling combined with the Zeeman term induce the topologically nontrivial superconducting state and indirectly give rise to substantial polarization of the Majorana modes (Figure 4), the oscillations of which show up near the chain edges (Figure 5). The polarized Majorana quasiparticles can also leak into other side-coupled objects, such as single or multiple quantum impurities (Figure 6). These polarized Majorana quasiparticles can be controlled by a magnetic field or by an electrostatic potential. This would be important for future quantum computers using qubits based on topologically protected Majorana states. Finally, we have also confronted the Majorana quasiparticles with the subgap Kondo effect, revealing their complex relationship that can be hardly regarded as competitive or collaborative in some analogy to the Kondo effect originating from multiple degrees of freedom [80]. The aforementioned spin-polarized effects can be experimentally verified by polarized ballistic tunneling or by using STM spectroscopy, relying on the selective equal-spin Andreev reflections.

\section{Acknowledgements}

We thank for instructive remarks from R. Aguado, J. Klinovaja, R. Lutchyn, P. Simon, and R. Žitko on different parts of our study. This work was supported by the National Science Centre (Poland) under grants DEC-2014/13/B/ST3/04451 (AK, SG, TD) and DEC-2013/11/B/ST3/00824 (MMM) UMO-2017/25/ 
B/ST3/02586 (AP) and by the Faculty of Mathematics and Natural Sciences of the University of Rzeszów through the project WMP/GD-06/2017 (GG).

\section{ORCID ${ }^{\circledR}$ iDs}

Andrzej Ptok - https://orcid.org/0000-0002-5566-2656

Maciej M. Maśka - https://orcid.org/0000-0003-2214-3283

\section{References}

1. Choi, S.; Choi, H. J.; Ok, J. M.; Lee, Y.; Jang, W.-J.; Lee, A. T.; Kuk, Y.; Lee, S.; Heinrich, A. J.; Cheong, S.-W.; Bang, Y.; Johnston, S.; Kim, J. S.; Lee, J. Phys. Rev. Lett. 2017, 119, 227001. doi:10.1103/PhysRevLett.119.227001

2. Kenzelmann, M.; Strässle, T.; Niedermayer, C.; Sigrist, M.; Padmanabhan, B.; Zolliker, M.; Bianchi, A. D.; Movshovich, R.; Bauer, E. D.; Sarrao, J. L.; Thompson, J. D. Science 2008, 321, 1652. doi:10.1126/science.1161818

3. Meul, H. W.; Rossel, C.; Decroux, M.; Fischer, Ø.; Remenyi, G.; Briggs, A. Phys. Rev. Lett. 1984, 53, 497. doi:10.1103/PhysRevLett.53.497

4. Balatsky, A. V.; Vekhter, I.; Zhu, J.-X. Rev. Mod. Phys. 2006, 78, 373. doi:10.1103/RevModPhys.78.373

5. Heinrich, B. W.; Pascual, J. I.; Franke, K. J. Prog. Surf. Sci. 2018, 93, 1. doi:10.1016/j.progsurf.2018.01.001

6. Yazdani, A.; Jones, B. A.; Lutz, C. P.; Crommie, M. F.; Eigler, D. M. Science 1997, 275, 1767. doi:10.1126/science.275.5307.1767

7. Ji, S.-H.; Zhang, T.; Fu, Y.-S.; Chen, X.; Ma, X.-C.; Li, J.; Duan, W.-H.; Jia, J.-F.; Xue, Q.-K. Phys. Rev. Lett. 2008, 100, 226801. doi:10.1103/PhysRevLett.100.226801

8. Franke, K. J.; Schulze, G.; Pascual, J. I. Science 2011, 332, 940. doi:10.1126/science.1202204

9. Ruby, M.; Pientka, F.; Peng, Y.; von Oppen, F.; Heinrich, B. W.; Franke, K. J. Phys. Rev. Lett. 2015, 115, 087001. doi:10.1103/PhysRevLett.115.087001

10. Hatter, N.; Heinrich, B. W.; Ruby, M.; Pascual, J. I.; Franke, K. J. Nat. Commun. 2015, 6, 8988. doi:10.1038/ncomms9988

11. Ménard, G. C.; Guissart, S.; Brun, C.; Pons, S.; Stolyarov, V. S.; Debontridder, F.; Leclerc, M. V.; Janod, E.; Cario, L.; Roditchev, D.; Simon, P.; Cren, T. Nat. Phys. 2015, 11, 1013. doi:10.1038/nphys3508

12. Jellinggaard, A.; Grove-Rasmussen, K.; Madsen, M. H.; Nygård, J. Phys. Rev. B 2016, 94, 064520. doi:10.1103/PhysRevB.94.064520

13. Choi, D.-J.; Rubio-Verdú, C.; de Bruijckere, J.; Ugeda, M. M.; Lorente, N.; Pascual, J. I. Nat. Commun. 2017, 8, 15175. doi:10.1038/ncomms 15175

14. Assouline, A.; Feuillet-Palma, C.; Zimmers, A.; Aubin, H.; Aprili, M.; Harmand, J.-C. Phys. Rev. Lett. 2017, 119, 097701. doi:10.1103/PhysRevLett.119.097701

15. Salkola, M. I.; Balatsky, A. V.; Schrieffer, J. R. Phys. Rev. B 1997, 55, 12648. doi:10.1103/PhysRevB.55.12648

16. Flatté, M. E.; Byers, J. M. Phys. Rev. Lett. 1997, 78, 3761. doi:10.1103/PhysRevLett.78.3761

17. Ugeda, M. M.; Bradley, A. J.; Zhang, Y.; Onishi, S.; Chen, Y.; Ruan, W.; Ojeda-Aristizabal, C.; Ryu, H.; Edmonds, M. T.; Tsai, H.-Z.; Riss, A.; Mo, S.-K.; Lee, D.; Zettl, A.; Hussain, Z.; Shen, Z.-X.; Crommie, M. F. Nat. Phys. 2016, 12, 92. doi:10.1038/nphys3527

18. Kezilebieke, S.; Dvorak, M.; Ojanen, T.; Liljeroth, P. Nano Lett. 2018, 18, 2311. doi:10.1021/acs.nanolett.7b05050
19. Santos, H.; Soriano, D.; Palacios, J. J. Phys. Rev. B 2014, 89, 195416. doi:10.1103/PhysRevB.89.195416

20. Kim, Y.; Zhang, J.; Rossi, E.; Lutchyn, R. M. Phys. Rev. Lett. 2015, 114, 236804. doi:10.1103/PhysRevLett.114.236804

21. Kaladzhyan, V.; Bena, C.; Simon, P. Phys. Rev. B 2016, 93, 214514. doi:10.1103/PhysRevB.93.214514

22. Ptok, A.; Głodzik, S.; Domański, T. Phys. Rev. B 2017, 96, 184425. doi:10.1103/PhysRevB.96.184425

23. Žitko, R.; Lim, J. S.; López, R.; Aguado, R. Phys. Rev. B 2015, 91 , 045441. doi:10.1103/PhysRevB.91.045441

24. Domański, T.; Weymann, I.; Barańska, M.; Górski, G. Sci. Rep. 2016, 6, 23336. doi:10.1038/srep23336

25. Smith, E. D. B.; Tanaka, K.; Nagai, Y. Phys. Rev. B 2016, 94, 064515. doi:10.1103/PhysRevB.94.064515

26. Goertzen, S. L.; Tanaka, K.; Nagai, Y. Phys. Rev. B 2017, 95, 064509. doi:10.1103/PhysRevB.95.064509

27. Koerting, V.; Andersen, B. M.; Flensberg, K.; Paaske, J. Phys. Rev. B 2010, 82, 245108. doi:10.1103/PhysRevB.82.245108

28. Pershoguba, S. S.; Björnson, K.; Black-Schaffer, A. M.; Balatsky, A. V. Phys. Rev. Lett. 2015, 115, 116602. doi:10.1103/PhysRevLett.115.116602

29. Głodzik, S.; Ptok, A. J. Supercond. Novel Magn. 2018, 31, 647. doi:10.1007/s10948-017-4360-6

30. Mashkoori, M.; Björnson, K.; Black-Schaffer, A. M. Sci. Rep. 2017, 7, 44107. doi:10.1038/srep44107

31. Satori, K.; Shiba, H.; Sakai, O.; Shimizu, Y. J. Phys. Soc. Jpn. 1992, 61, 3239-3254. doi:10.1143/JPSJ.61.3239

32. Sakai, O.; Shimizu, Y.; Shiba, H.; Satori, K. J. Phys. Soc. Jpn. 1993 62, 3181-3197. doi:10.1143/JPSJ.62.3181

33. Sakurai, A. Prog. Theor. Phys. 1970, 44, 1472. doi:10.1143/PTP.44.1472

34. van Gerven Oei, W.-V.; Tanasković, D.; Žitko, R. Phys. Rev. B 2017, 95, 085115. doi:10.1103/PhysRevB.95.085115

35. Morr, D. K.; Yoon, J. Phys. Rev. B 2006, 73, 224511. doi:10.1103/PhysRevB.73.224511

36. Röntynen, J.; Ojanen, T. Phys. Rev. Lett. 2015, 114, 236803. doi:10.1103/PhysRevLett.114.236803

37. Morr, D. K.; Stavropoulos, N. A. Phys. Rev. B 2003, 67, 020502. doi:10.1103/PhysRevB.67.020502

38. Kawakami, T.; Hu, X. Phys. Rev. Lett. 2015, 115, 177001. doi:10.1103/PhysRevLett.115.177001

39. Nadj-Perge, S.; Drozdov, I. K.; Li, J.; Chen, H.; Jeon, S.; Seo, J.; MacDonald, A. H.; Bernevig, B. A.; Yazdani, A. Science 2014, 346, 602. doi:10.1126/science.1259327

40. Pawlak, R.; Kisiel, M.; Klinovaja, J.; Maier, T.; Kawai, S.; Glatzel, T.; Loss, D.; Meyer, E. npj Quantum Inf. 2016, 2, 16035. doi:10.1038/npjqi.2016.35

41. Ruby, M.; Pientka, F.; Peng, Y.; von Oppen, F.; Heinrich, B. W.; Franke, K. J. Phys. Rev. Lett. 2015, 115, 197204. doi:10.1103/PhysRevLett.115.197204

42. Jeon, S.; Xie, Y.; Li, J.; Wang, Z.; Bernevig, B. A.; Yazdani, A. Science 2017, 358, 772. doi:10.1126/science.aan3670

43. Maśka, M. M.; Gorczyca-Goraj, A.; Tworzydło, J.; Domański, T. Phys. Rev. B 2017, 95, 045429. doi:10.1103/PhysRevB.95.045429

44. Liu, X.; Li, X.; Deng, D.-L.; Liu, X.-J.; Das Sarma, S. Phys. Rev. B 2016, 94, 014511. doi:10.1103/PhysRevB.94.014511

45. Stanescu, T. D.; Tewari, S. J. Phys.: Condens. Matter 2013, 25 , 233201. doi:10.1088/0953-8984/25/23/233201

46. Sticlet, D.; Bena, C.; Simon, P. Phys. Rev. Lett. 2012, 108, 096802. doi:10.1103/PhysRevLett.108.096802 
47. Maśka, M. M.; Domański, T. Sci. Rep. 2017, 7, 16193. doi:10.1038/s41598-017-16323-3

48. Vernek, E.; Penteado, P. H.; Seridonio, A. C.; Egues, J. C. Phys. Rev. B 2014, 89, 165314. doi:10.1103/PhysRevB.89.165314

49. Deng, M. T.; Vaitiekenas, S.; Hansen, E. B.; Danon, J.; Leijnse, M.; Flensberg, K.; Nygård, J.; Krogstrup, P.; Marcus, C. M. Science 2016, 354, 1557. doi:10.1126/science.aaf3961

50. Liu, C.-X.; Sau, J. D.; Stanescu, T. D.; Das Sarma, S. Phys. Rev. B 2017, 96, 075161. doi:10.1103/PhysRevB.96.075161

51. Hoffman, S.; Chevallier, D.; Loss, D.; Klinovaja, J. Phys. Rev. B 2017, 96, 045440. doi:10.1103/PhysRevB.96.045440

52. Ptok, A.; Kobiałka, A.; Domański, T. Phys. Rev. B 2017, 96, 195430. doi:10.1103/PhysRevB.96.195430

53. Chevallier, D.; Szumniak, P.; Hoffman, S.; Loss, D.; Klinovaja, J. Phys. Rev. B 2018, 97, 045404. doi:10.1103/PhysRevB.97.045404

54. Chirla, R.; Moca, C. P. Phys. Rev. B 2016, 94, 045405. doi:10.1103/PhysRevB.94.045405

55. Prada, E.; Aguado, R.; San-Jose, P. Phys. Rev. B 2017, 96, 085418. doi:10.1103/PhysRevB.96.085418

56. Barański, J.; Kobiałka, A.; Domański, T. J. Phys.: Condens. Matter 2017, 29, 075603. doi:10.1088/1361-648X/aa5214

57. Domański, T.; Žonda, M.; Pokorný, V.; Górski, G.; Janiš, V.; Novotný, T. Phys. Rev. B 2017, 95, 045104. doi:10.1103/PhysRevB.95.045104

58. Mourik, V.; Zuo, K.; Frolov, S. M.; Plissard, S. R.; Bakkers, E. P. A. M.; Kouwenhoven, L. P. Science 2012, 336, 1003. doi:10.1126/science.1222360

59. Gül, Ö.; Zhang, H.; Bommer, J. D. S.; de Moor, M. W. A.; Car, D.; Plissard, S. R.; Bakkers, E. P. A. M.; Geresdi, A.; Watanabe, K.; Taniguchi, T.; Kouwenhoven, L. P. Nat. Nanotechnol. 2018, 13, 192. doi:10.1038/s41565-017-0032-8

60. Golub, A. Phys. Rev. B 2015, 91, 205105. doi:10.1103/PhysRevB.91.205105

61. Bauer, J.; Oguri, A.; Hewson, A. C. J. Phys.: Condens. Matter 2007, 19, 486211. doi:10.1088/0953-8984/19/48/486211

62. Yamada, Y.; Tanaka, Y.; Kawakami, N. Phys. Rev. B 2011, 84, 075484. doi:10.1103/PhysRevB.84.075484

63. Martín-Rodero, A.; Levy Yeyati, A. Adv. Phys. 2011, 60, 899. doi:10.1080/00018732.2011.624266

64. Barański, J.; Domański, T. J. Phys.: Condens. Matter 2013, 25, 435305. doi:10.1088/0953-8984/25/43/435305

65. Liu, D. E.; Cheng, M.; Lutchyn, R. M. Phys. Rev. B 2015, 91, 081405. doi:10.1103/PhysRevB.91.081405

66. Elliott, S. R.; Franz, M. Rev. Mod. Phys. 2015, 87, 137. doi:10.1103/RevModPhys.87.137

67. Ruiz-Tijerina, D. A.; Vernek, E.; Dias da Silva, L. G. G. V.; Egues, J. C. Phys. Rev. B 2015, 91, 115435. doi:10.1103/PhysRevB.91.115435

68. Lee, M.; Lim, J. S.; López, R. Phys. Rev. B 2013, 87, 241402. doi:10.1103/PhysRevB.87.241402

69. Cheng, M.; Becker, M.; Bauer, B.; Lutchyn, R. M. Phys. Rev. X 2014, 4, 031051. doi:10.1103/PhysRevX.4.031051

70. van Beek, I. J.; Braunecker, B. Phys. Rev. B 2016, 94, 115416. doi:10.1103/PhysRevB.94.115416

71. Weymann, I. J. Phys.: Condens. Matter 2017, 29, 095301. doi:10.1088/1361-648X/aa5526

72. Weymann, I.; Wójcik, K. P. Phys. Rev. B 2017, 95, 155427. doi:10.1103/PhysRevB.95.155427

73. López, R.; Lee, M.; Serra, L.; Lim, J. S. Phys. Rev. B 2014, 89, 205418. doi:10.1103/PhysRevB.89.205418
74. Tanaka, Y.; Kawakami, N.; Oguri, A. J. Phys. Soc. Jpn. 2007, 76, 074701. doi:10.1143/JPSJ.76.074701

75. Vecino, E.; Martín-Rodero, A.; Levy Yeyati, A. Phys. Rev. B 2003, 68, 035105. doi:10.1103/PhysRevB.68.035105

76. Béri, B.; Cooper, N. R. Phys. Rev. Lett. 2012, 109, 156803. doi:10.1103/PhysRevLett.109.156803

77. Galpin, M. R.; Mitchell, A. K.; Temaismithi, J.; Logan, D. E.; Béri, B.; Cooper, N. R. Phys. Rev. B 2014, 89, 045143. doi:10.1103/PhysRevB.89.045143

78. Plugge, S.; Zazunov, A.; Eriksson, E.; Tsvelik, A. M.; Egger, R. Phys. Rev. B 2016, 93, 104524. doi:10.1103/PhysRevB.93.104524

79. Béri, B. Phys. Rev. Lett. 2017, 119, 027701. doi:10.1103/PhysRevLett.119.027701

80. Jacob, D.; Soriano, M.; Palacios, J. J. Phys. Rev. B 2013, 88, 134417. doi:10.1103/PhysRevB.88.134417

\section{License and Terms}

This is an Open Access article under the terms of the Creative Commons Attribution License (http://creativecommons.org/licenses/by/4.0), which permits unrestricted use, distribution, and reproduction in any medium, provided the original work is properly cited.

The license is subject to the Beilstein Journal of

Nanotechnology terms and conditions:

(https://www.beilstein-journals.org/bjnano)

The definitive version of this article is the electronic one which can be found at: $\underline{\text { doi: } 10.3762 / \text { bjnano.9.129 }}$ 